Research Article

\title{
Distributed Intrusion Sensor Using DFB Laser with Optical Feedback and Saturable Absorber
}

\author{
Kyoo Nam Choi \\ Department of Information and Telecommunication Engineering, Incheon National University, Incheon 22012, Republic of Korea \\ Correspondence should be addressed to Kyoo Nam Choi; knchoi@inu.ac.kr
}

Received 18 September 2017; Revised 14 December 2017; Accepted 26 December 2017; Published 8 February 2018

Academic Editor: Stefano Stassi

Copyright (C) 2018 Kyoo Nam Choi. This is an open access article distributed under the Creative Commons Attribution License, which permits unrestricted use, distribution, and reproduction in any medium, provided the original work is properly cited.

\begin{abstract}
Characteristics of a distributed intrusion sensor using a coherent DFB laser diode with an external optical feedback and saturable absorber were experimentally investigated. The stimulus at a location of $2 \mathrm{~km}$ using a PZT transducer placed the location of a simulated intruder in $\Phi$-OTDR trace after averaging 32 times. Field trials demonstrated the detection of a vehicle and a pedestrian crossing above the sensing line and a loop in a burial depth of $50 \mathrm{~cm}$. This distributed intrusion sensor using a coherent DFB laser diode as the light source had the advantages of a simple structure and intruder detection capability at the underground burial location.
\end{abstract}

\section{Introduction}

The intrusion sensors to locate an intruder's position are drawing increasing attention in the security industry. However, it requires multiple point sensors to extend the detection range using conventional point sensors [1]. Thus, it has been desired to detect an intruder with location information using a single sensor. For this purpose, the distributed sensor became the proper choice $[2,3]$. Also, it is necessary to conceal the intrusion sensor in an unexposed location, such as buried underground [4]. The sensitivity of a sensor decreases considerably as the burial depth increases [5]; thus, the sensitivity needs to compensate for the attenuation due to absorption in soil. In this respect, the coherent fiber optical sensor is a suitable candidate in terms of sensitivity [6]. However, the resolution of a coherent optical sensor depends on the coherence length of a light source [7]. Various concepts have been proposed regarding intrusion sensors. A single intrusion sensor using a leaky coaxial cable has been demonstrated to provide intrusion location along a perimeter sensor cable with shallow burial [8], and the coupled wave device (CWD) using a perimeter guided radar detection system has shown the capability to detect over complex geometry above ground [9]. However, these wired sensing methods have a relatively short detection range, below $\mathrm{km}$ scale, due to attenuation in copper wire. In the case of using multimode optical fiber, an intrusion sensor using Speckle pattern variation showed moderate sensitivity, but without location information [10]. The single-mode optical fiber intrusion sensor using Mach-Zehnder interferometry [11] or fiber Bragg grating (FBG) showed exceptional sensitivity, but also without intruder location information. Thus, multiple sensors were required using cascaded or array architecture to provide intruder location information. The intrusion sensing technique using Rayleigh scattering in a single-mode optical fiber had the advantage of providing distance information; however, the sensitivity in optical time domain reflectometry (OTDR) configuration [12] was not sufficient for underground burial application. The phase-sensitive $\Phi$-OTDR had fully distributed extremely high sensitivity with accurate localization and simultaneous multipoint detection capability in comparison to other optical fiber intrusion sensors. However, it used a fiber laser with an internal active medium [13], which is exposed to ambient air. And the ambient factors such as airflow, vibration, and temperature inherently influenced the stability of the coherent laser. Thus, the optical fiber intrusion sensor with a compact coherent light source with an integrated active medium has been preferred for outdoor field intrusion sensor application. Our present interest is to realize an optical fiber intrusion sensor with a 
compact coherent light source, which is immune to ambient factors in comparison to a coherent fiber laser, for field application.

This paper is about a distributed phase-sensitive $\Phi$ OTDR intrusion sensing system with a compact coherent light source using a distributed feedback semiconductor laser with an optical feedback and a saturable absorber.

\section{Distributed Optical Fiber Sensor}

2.1. Coherent $\Phi-O T D R$. The architecture of coherent $\Phi$ OTDR is similar to conventional OTDR for locating fault points of communication optical fiber. The difference is that the coherent $\Phi$-OTDR requires a light source with a longer coherence length and narrower linewidth. The light from a coherent light source is modulated using an external modulator, and it is converted to continuous short optical pulse trains. Then, these light pulse trains are optically amplified to transmit through a long-haul optical fiber sensing line. The narrow light pulse from an optical fiber amplifier is divided by a $3 \mathrm{~dB}$ optical fiber coupler to transmit an optical probe pulse and to receive the returned sensing signal simultaneously. The optical sensing signal returned by Rayleigh backscattering is converted into an electrical waveform in a PIN photodetector. The single-mode optical fiber for optical communication is used to sense an intruder. Then, the electrical signal is amplified and filtered to enhance the signal-to-noise ratio. Furthermore, the signal is averaged to reduce jagged waveform and processed to deduce the intruder location information. The concept of coherent $\Phi-O T D R$ to discriminate the presence of an intruder is shown in Figure 1.

In case where the light pulse travels the distance through an optical fiber core, a part of the light is scattered by volume having a refractive index difference in comparison to the refractive index of the optical fiber core. If the incident light intensity is $I_{i}$, then the total scattered light intensity $I_{s}$, which is reflected by $j$ number of scattering objects, can be estimated $[14,15]$. The ratio, $R_{b s}$, of scattered light power, $\Delta P$, per incident light power, $P(z)$, at distance $z$ in a single-mode fiber can be approximately estimated, as shown in (1), because the attenuation of optical fiber is mostly due to Rayleigh scattering. In (1), $F$ represents the ratio of backscattered light when incident optical power has the Gaussian, and $\alpha$ is the attenuation coefficient [16].

$$
R_{b s} \approx F \cdot\left|\frac{\Delta P}{P(z)}\right|=F \cdot \alpha \cdot \Delta z .
$$

The distance, $L$, to the intruder location is calculated by the time difference between transmitted, $T_{T}$, and received, $T_{R}$, light pulses using group velocity, $v_{g}$, of light inside the sensing fiber core, as shown in (2). Also, the distance resolution, $\Delta z$, depends on pulse width, $T_{p w}$, of the transmitted light pulse, as shown in (3). The received power, $P_{b s}$, by backscattering at distance $L$ from the transmitter, can be expressed as shown in (4), when the incident optical power is $P_{i}$. Thus, the sensitivity of a distributed optical fiber sensor is proportional to transmitted optical power and pulse width. If we decrease

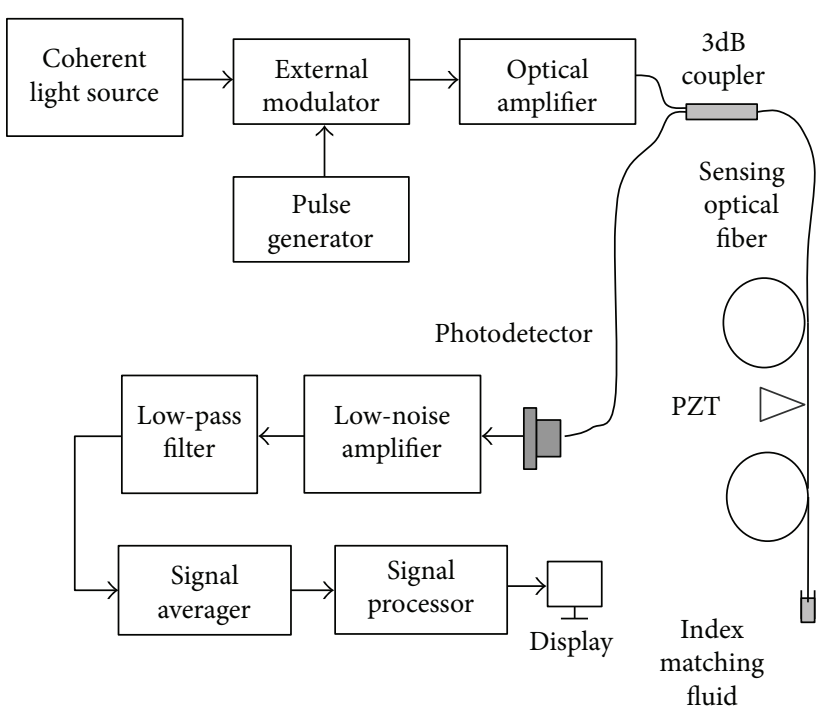

FIgURE 1: The block diagram of coherent $\Phi$-OTDR distributed sensor.

the pulse width to increase the distance resolution, it will result in decreased signal reception. Thus, an optical power amplifier is essential in a distributed intrusion sensor.

$$
\begin{aligned}
L & =v_{g} \cdot \frac{T_{R}-T_{T}}{2}, \\
\Delta z & \approx \frac{T_{p w} v_{g}}{2}, \\
P_{b s} & \approx \frac{1}{2} \cdot F \cdot \alpha \cdot v_{g} \cdot T_{p w} \cdot P_{i} \cdot e^{-2 \alpha L} .
\end{aligned}
$$

The coherent $\Phi$-OTDR has a significant advantage over conventional OTDR in terms of sensitivity; however, it requires the light source to have a narrow linewidth as well as low frequency drift. The backscattered light wave in coherent $\Phi$-OTDR forms a random state of polarization; thus, the reception signal shows a jagged waveform. The intruder induces the phase modulation to backscattered signal inside the optical fiber core, and this phase modulated signal can be expressed as a nonstationary autocorrelation function, $R_{y y}$, as shown in

$$
R_{y y}(t, t-T)=E\left[y_{i}(t) \cdot y_{j}(t-T)\right] .
$$

The envelop of a phase modulated signal shows Rayleigh distribution, because $y(t)$ is a narrowband noise process [17]. The jagged waveform due to Rayleigh distribution and fading can be reduced by filtering and averaging when the variation is within a tolerable range. Ambient factors, such as vibration and instant temperature variation due to airflow, influence phase stability of the light source, because they modulate the optical phase inside the optical fiber core. The performance of an intrusion sensor using a $\Phi$-OTDR configuration requires not only longer coherence length but also immunity to ambient factors, and those are directly related to measurement precision. 


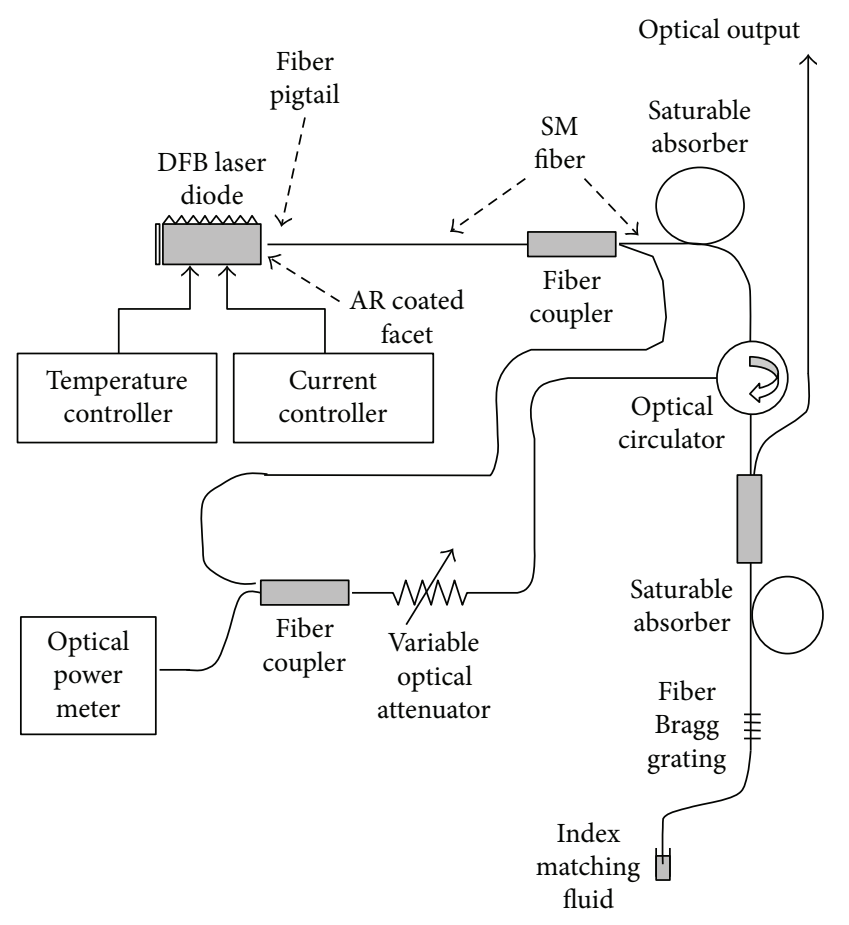

FIgURE 2: The block diagram of coherent light source.

2.2. Coherent Light Source. Spectral linewidth is an important parameter of a fiber-optic distributed intrusion sensor. The linewidth requirement for coherent optical communication is less than $500 \mathrm{kHz}$ [18] and tens of $\mathrm{kHz}$ for fiber-optic gyro application [19]. The fiber-optic intrusion sensor showed distributed sensing capability using a fiber laser with a $<3 \mathrm{kHz}$ spectral linewidth [7]. The experimental spectral linewidth value of an $\mathrm{AlGaAs} / \mathrm{GaAs}$ CSP laser is $\sim 38 \mathrm{MHz}$ at $3 \mathrm{~mW}$ [20]. A distributed feedback (DFB) laser has an active region with a periodic structure as a diffraction grating, and this grating behaves not only as a wavelength selection element but also as a resonator to produce a single longitudinal mode, in contrast to a conventional Fabry-Perot laser. A conventional single-frequency tunable DFB laser diode has a spectral linewidth of $\sim 2 \mathrm{MHz}$, and this is regarded as too broad for fiber-optic distributed sensor application. A longer laser cavity and higher facet reflectivity are needed for narrow linewidth [21]; however, this leads to laser mode hopping and multilongitudinal mode oscillation in case of a Fabry-Perot cavity. The linewidth $\Delta v$ of the tunable DFB laser diode is approximate as shown in (6), where $\alpha_{0}$ is the sum of absorption loss and scattering loss, $\kappa$ is the coupling coefficient, and $L$ is the cavity length [22]. This shows that the higher $\kappa$ and longer $L$ are needed to shorten spectral linewidth. Also, the external optical feedback to a DFB laser diode affects the linewidth of the laser [23]. The absorption coefficient varies according to the absorbed light quantity in a saturable absorber [24], and this can affect the spectral linewidth in the lasing cavity.

$$
\Delta v \propto \frac{\alpha_{0}}{\kappa^{2} L^{3}}
$$

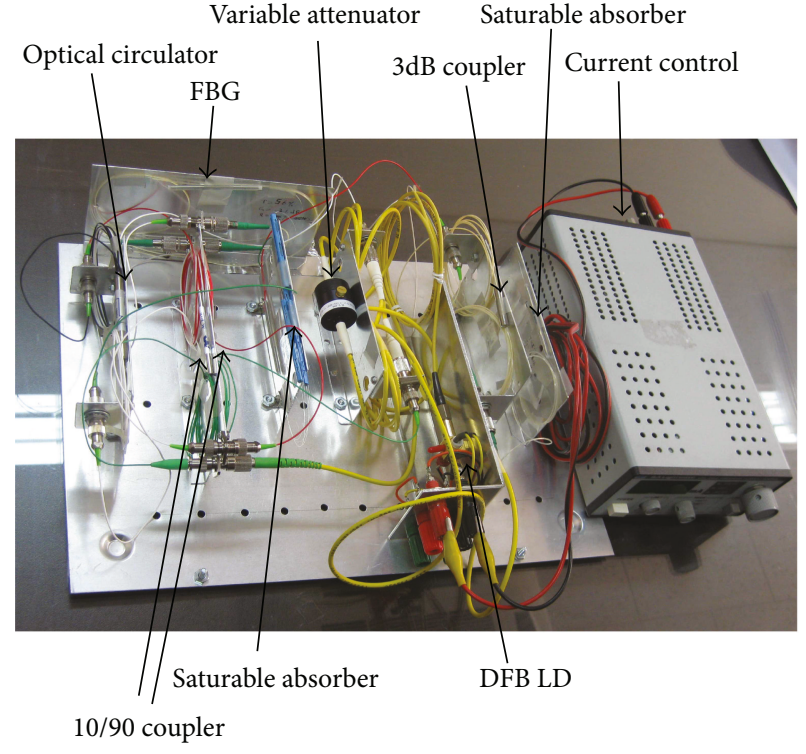

FIgURE 3: Top view of coherent light source.

A coherent light source is fabricated using a DFB laser diode, antireflection coated on one facet side and highreflection on the other facet side to form an external optical cavity between an external fiber Bragg grating and DFB laser diode. An optical feedback loop is formed by using an optical circulator. The amount of optical feedback is adjusted by using a variable optical attenuator, and it is monitored using an optical fiber coupler. The center wavelength of the external fiber Bragg grating is selected to match the lasing wavelength of the DFB laser diode. Furthermore, the lasing wavelength of the DFB laser diode is precisely adjusted to the center wavelength of the external fiber Bragg grating by adjusting the temperature of the DFB laser diode. The unconnected fiber end is immersed in index matching fluid to avoid unwanted reflection. Two saturable absorbers are inserted in the lasing cavity to further reduce the spectral linewidth, as shown in Figure 2. The erbium-doped optical fiber is used as the saturable absorber.

2.3. Spectral Linewidth Measurement. The wavelength resolution of a conventional optical spectrum analyzer is $\sim 0.1 \mathrm{~nm}$; thus, a self-heterodyne measurement setup is needed to measure the extremely narrow linewidth [25]. The resolution of the self-heterodyne measurement setup is determined by optical fiber delay line length, L, where the delay time, $\tau_{\text {delay }}=n_{g} / c$, is $\sim 5 \mu \mathrm{s} / \mathrm{km} \times \mathrm{L} \mathrm{km}$. In the equation, $c$ is the speed of light, and $n_{g}$ is the group refractive index. The free spectral range (FSR), $\Delta v_{\mathrm{FSR}}$, is represented as $c /\left(n_{g} L_{c}\right)$, where $L_{c}$ is the coherence length. Thus, the spectral width exceeding $\Delta v_{\mathrm{FSR}}$ can be measured within the resolution limit. The $-3 \mathrm{~dB}$ spectral width, $\Delta \lambda_{\mathrm{FWHM}}$, of a light source can be calculated from coherence length using $\lambda_{\mathrm{o}}^{2} /\left(\pi L_{c}\right)$ [26]. An optical modulator can be used to shift the center frequency to display the spectral waveform on a RF spectrum analyzer. 


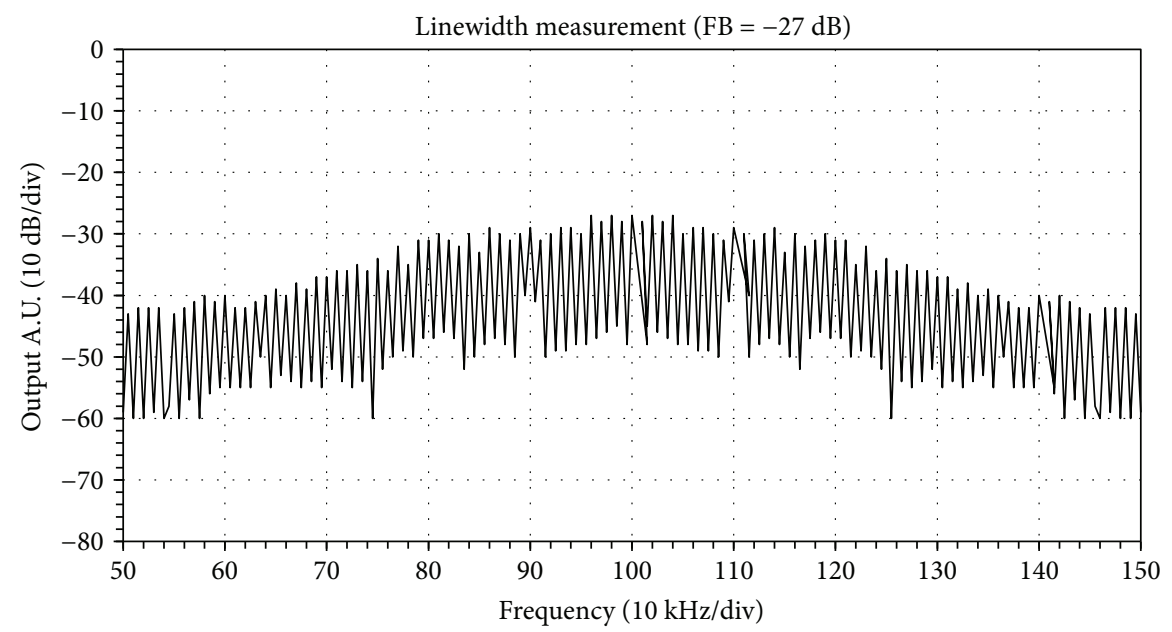

FIGURE 4: RF spectrum of self-heterodyne setup, when $P_{\mathrm{FB}}=-27 \mathrm{~dB}$.

\section{Experiment}

3.1. Coherent Light Source. The DFB laser diode with $\lambda c=1530.618 \mathrm{~nm}$ center wavelength and $\Delta \lambda=0.02 \mathrm{~nm}$ wavelength width was used as the pumping source. An FBG with $\lambda c=1530.32 \mathrm{~nm}$ center wavelength at $23.5^{\circ} \mathrm{C}$ and $\Delta \lambda=0.99 \mathrm{~nm}$ wavelength width was used as the reflector to form an external Fabry-Perot cavity. The lasing wavelength of the DFB laser diode was adjusted to the center wavelength of the FBG by controlling the temperature of the DFB laser diode. The exact matching of two center wavelengths is essential to the performance of a coherent light source. Two erbium-doped fibers, each 1-inch long, having $-7.5 \mathrm{~dB} / \mathrm{m}$ loss at $1530 \mathrm{~nm}$, were used as saturable absorbers. Also, a variable optical attenuator with an attenuation range of $0 \sim 60 \mathrm{~dB}$ was used to provide optical feedback. The optical power of the coherent light source with an external Fabry-Perot cavity was reduced to $\sim 5 \%$ of the DFB laser diode alone. The assembled coherent light source is shown in Figure 3.

3.2. Spectral Linewidth Measurement. In self-heterodyne measurement setup, the phase and frequency fluctuation of an incident optical signal is converted to an amplitude signal, and it is measured using a RF spectrum analyzer. In the Mach-Zehnder interferometer configuration of a selfheterodyne measurement setup, the incident optical signal meets its identical signal through a fiber-optic delay line. At this time, two signals interfere with each other and are converted from linewidth information to RF power spectrum. The carrier frequency $\omega_{c}, 100 \mathrm{kHz}$ in this experiment, was applied to one side of the fiber-optic line to shift the center frequency of the interference signal for viewing in a RF spectrum analyzer. In the self-heterodyne measurement setup, if the length of a fiber-optic delay line is longer, the wavelength resolution becomes better. At the same time, the optical signal power through the fiber-optic delay line needs to be above the noise equivalent power (NEP) value of an optical receiver. When the length of a fiber-optic delay line is equal or shorter than the linewidth of a light source, the phase of the interference signal becomes partially correlated. The fiber-optic delay line length of the self-heterodyne measurement setup was selected as $63 \mathrm{~km}$, which is equivalent to a $\sim 0.315 \mathrm{~ms}$ time delay.

In case of no optical feedback, the linewidth will be identical to the linewith of the long cavity DFB laser diode. In the case of excess optical feedback, instability will be increased due to spatial hole burning (SHB) and mode hopping. Thus, it is necessary to adjust the amount of optical feedback to achieve the minimum linewidth. In the first experiment, the optical feedback, $P_{\mathrm{FB}}$, was adjusted to $-27 \mathrm{~dB}$ of optical output power, and the RF spectrum of the self-heterodyne measurement setup is shown in Figure 4. In the second experiment, the amount of optical feedback, $P_{\mathrm{FB}}$, was increased to $-17 \mathrm{~dB}$ of optical output power without the two saturable absorbers. In this case, the linewidth was enhanced, as shown in Figure 5. In the third experiment, two saturable absorbers were added to the second case, as shown in Figure 2, and then the measured linewidth showed better enhancement, as shown in Figure 6 .

The coherent light source with two saturable absorbers had the most narrow linewidth among the three measurements. The measured $-3 \mathrm{~dB}$ linewidth in Figure 6 is $\sim 3 \mathrm{kHz}$, which is the resolution limit of the self-heterodyne measurement setup. The actual linewidth is considered to be below $3 \mathrm{kHz}$. The $3 \mathrm{kHz}$ linewidth is the FSR, $\Delta v_{\mathrm{FSR}}=$ $c /\left(n_{g} L_{c}\right)$, which is equivalent to $\sim 1 \times 10^{-8} \mathrm{~nm}$ using $\Delta \lambda_{\mathrm{FWHM}}=\lambda_{\mathrm{o}}^{2} /\left(\pi L_{c}\right)$.

3.3. Coherent OTDR. The coherent light source was encased to isolate it from an outside airflow effect. It was doublewrapped with cotton wool and sponge in a styrofoam case. The light from the coherent light source was amplified $30 \mathrm{~dB}$ using an optical amplifier. Two cascaded optical single-mode fiber spools, each $2 \mathrm{~km}$ and $10 \mathrm{~km}$ length, were used as simulated sensing lines. The optical fiber spools were encased in similar shock absorbing and temperature-stable cases. The dimensions of the case were $\mathrm{L} 60 \times \mathrm{D} 60 \times \mathrm{H} 60 \mathrm{~cm}$. The PZT (lead zirconate titanate) was used to simulate the intruder and located between $2 \mathrm{~km}$ fiber spool and $10 \mathrm{~km}$ 


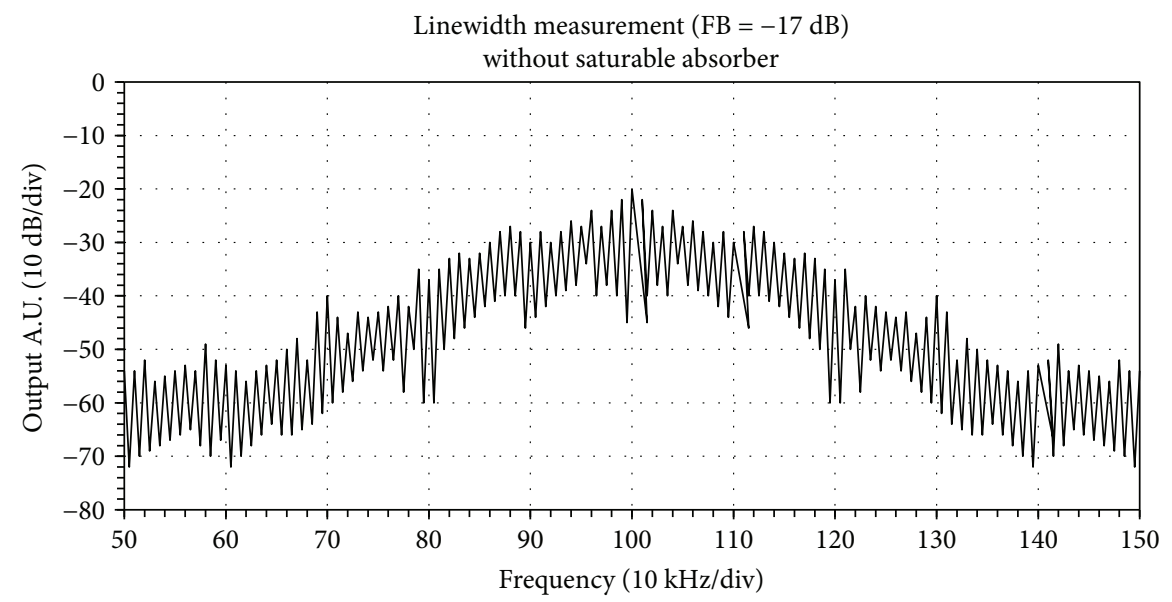

Figure 5: RF spectrum of self-heterodyne setup, without saturable absorber, when $P_{\mathrm{FB}}=-17 \mathrm{~dB}$.

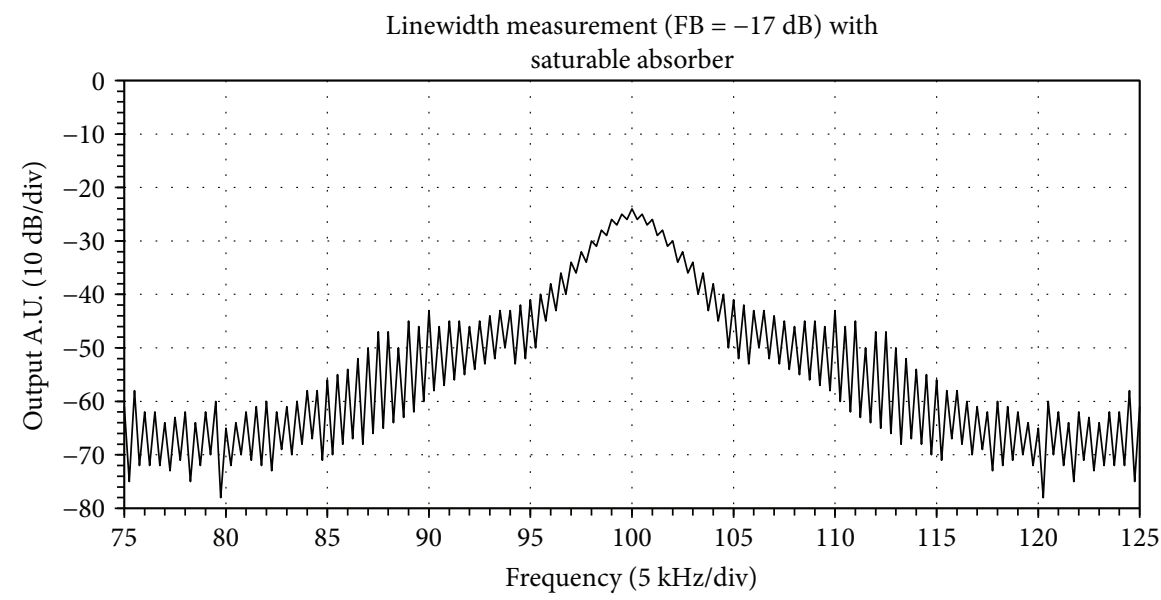

Figure 6: RF spectrum of self-heterodyne setup, with saturable absorbers, when $P_{\mathrm{FB}}=-17 \mathrm{~dB}$.

fiber spool. The phase modulation of the PZT was adjusted to $2 / 3$ level of maximum output. The $3 \mathrm{~dB}$ optical fiber coupler was used to transmit and receive optical signals simultaneously. The signal received was amplified $33 \mathrm{~dB}$ using lownoise amplifier (LNA) and filtered by using low-pass filter (LPF) with variable cutoff frequency capability. Finally, the signal was averaged to minimize signal fluctuation. The experimental setup for measurement is shown in Figure 7.

The peak signal at the $2 \mathrm{~km}$ location from the starting point in the $12 \mathrm{~km}$-long sensor line showed the sensing of the simulated intruder by the PZT transducer. In the $\Phi-O T D R$ trace, as shown in Figure 8 , the $40 \mu$ s point is the starting location, and the $60 \mu$ s point is the $2 \mathrm{~km}$ location. The elapsed time of $20 \mu$ s is equivalent to the round-trip time through the $2 \mathrm{~km}$ fiber-optic sensor line. Vehicle traffic outside the building and pedestrians walking inside the building both behaved as environmental noise, even though the optical fiber spools were contained in shock absorbing boxes. Thus, the measurement was performed at midnight when there was no vehicle traffic or pedestrians walking. The noise-like waveform in Figure 8(b) is due partly to the frequency instability of the light source and partly to environmental noise sources.

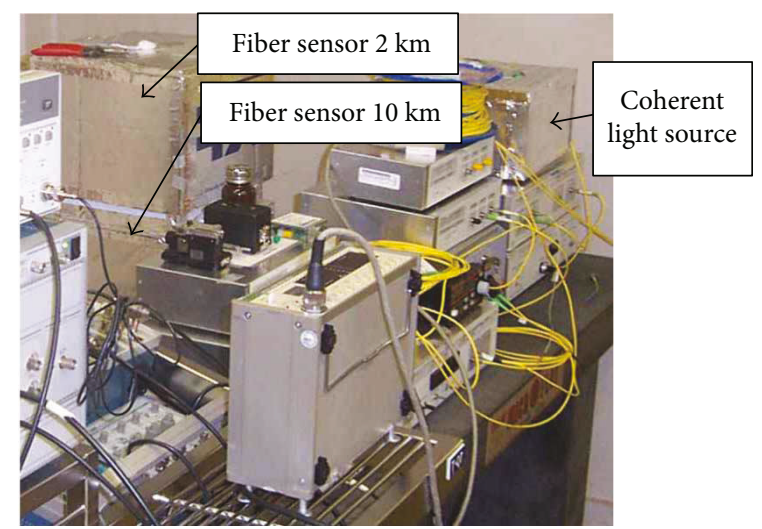

FIGURE 7: Experimental setup of distributed intrusion sensor.

3.4. Intrusion Sensor. A field test was conducted to evaluate the performance of the intrusion sensor. A single-mode optical fiber of optical communication grade was used as the sensing line. The sensing line, with a total length of $100 \mathrm{~m}$ with a $3 \mathrm{~mm}$ diameter jacket, $44 \mathrm{~m}$ as buried and $56 \mathrm{~m}$ as an outside connecting line, was inserted at a location 


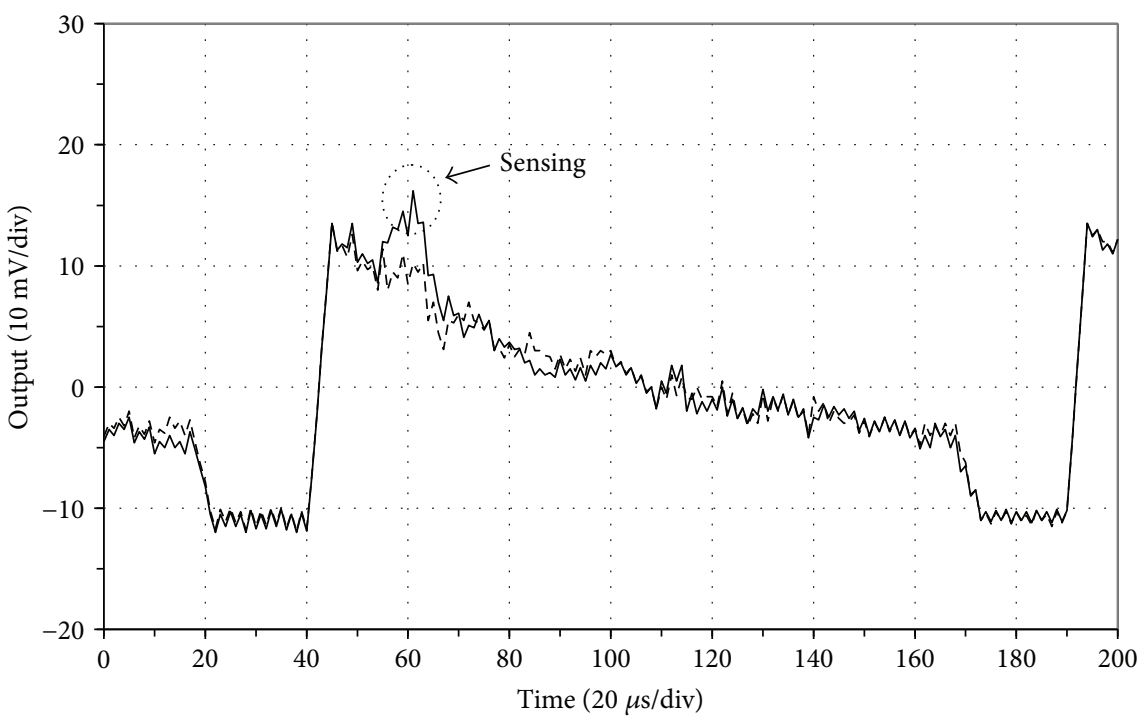

(a)

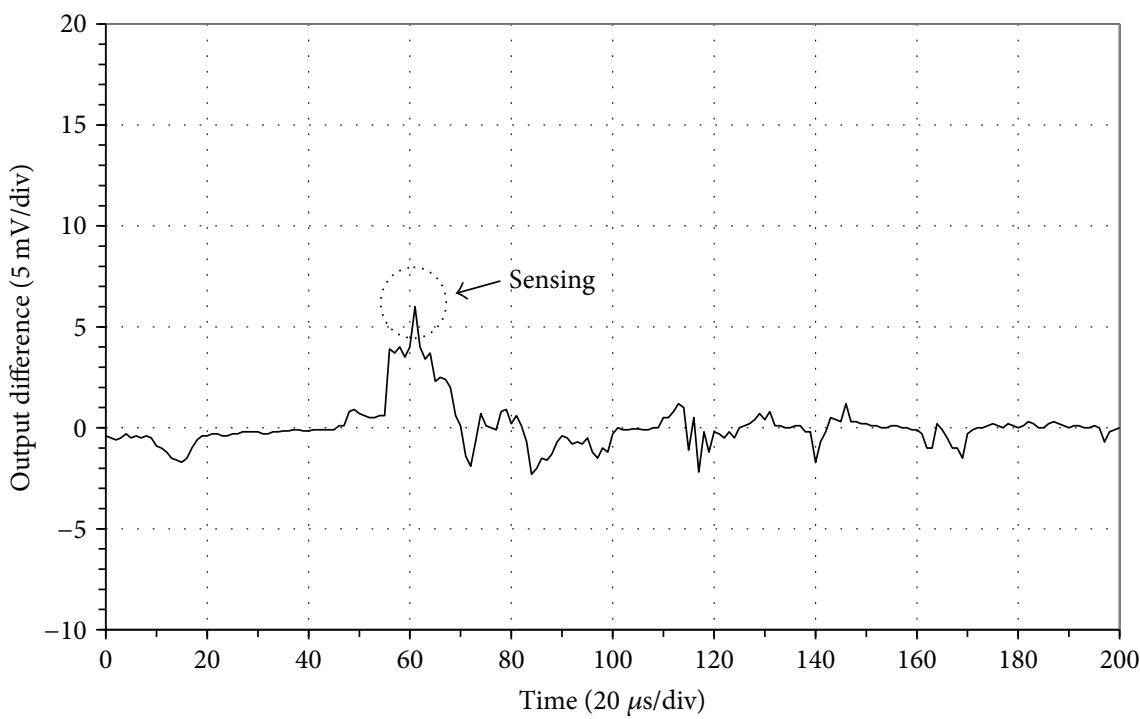

(b)

FIgURE 8: Ф-OTDR trace of distributed intrusion sensor. (a) Waveform before detection (dot) and after detection (line). (b) Difference waveform.

$2 \mathrm{~km}$ from the light source and $12 \mathrm{~km}$ in total length. The sensing line was buried at a depth of $50 \mathrm{~cm}$ and backfilled with soil. The burial layout of the fiber-optic sensing cable is shown in Figure 9(a), and a photo showing the backfilled surface is shown in Figure 9(b). A vehicle weighing $\sim 1500 \mathrm{~kg}$ and a pedestrian weighing $\sim 60 \mathrm{~kg}$ were used as sample intruders. The test was carried out for two cases: one crossing the whole sensing loop and the other crossing one sensing line only.

In case of crossing one sensing line, the detection response for the vehicle created notches around the sensing peak, while the response for the pedestrian showed the one dominant peak. The phase-sensitive $\Phi$-OTDR traces for these two cases, after averaging 32 times, are shown in Figures 10 and 11. By comparing the traces between Figures 10(b) and 11(b), the peak output values were found

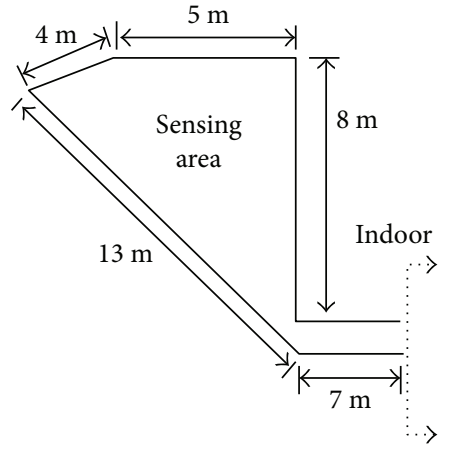

(a)

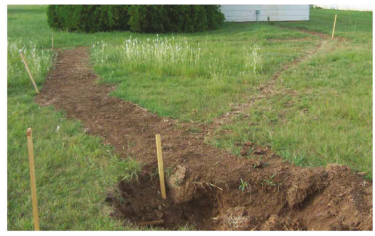

(b)
FIgURE 9: Layout of buried sensing cable. (a) Dimension. (b) Surface soil. 


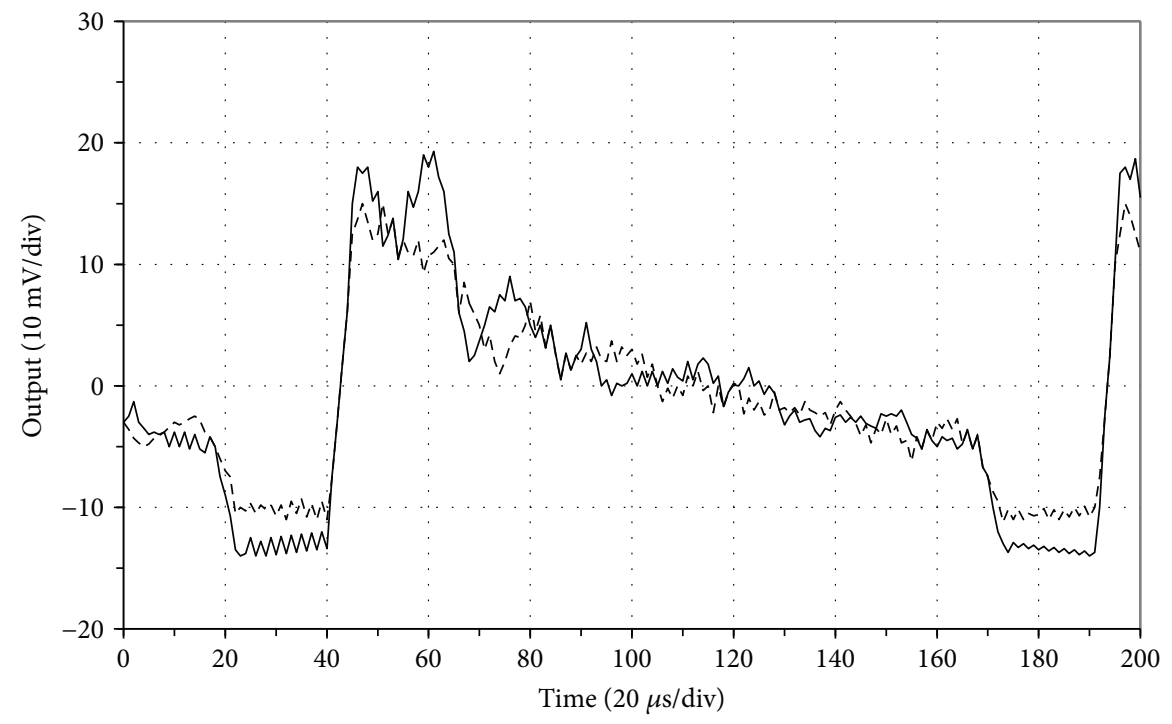

(a)

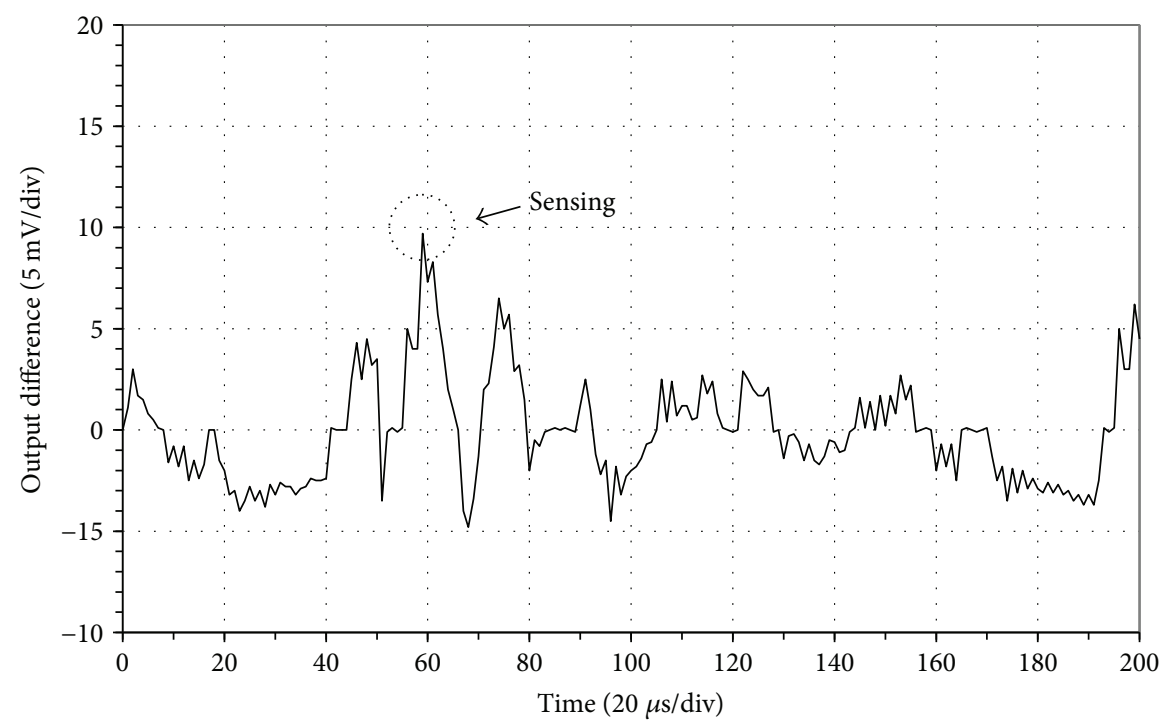

(b)

FIgure 10: Phase-sensitive OTDR trace by vehicle in case of crossing one sensing line. (a) Waveform before detection (dot) and after detection (line). (b) Difference waveform.

to be similar. This can be explained as both phase shifts, by vehicle and pedestrian, were more than $2 \pi$ rad., which was enough to be detected. The difference between Figures 10(b) and 11(b) indicates that the impact of the vehicle was strong enough to influence both optical fiber spools, which are $12 \mathrm{~km}$ long, even though the spools were contained in shock resistance containers. However, one momentary movement by the pedestrian located the intruder at the $2 \mathrm{~km}$ location. The noise-like waveform in the other location, as seen in Figure 11(b), is due to frequency instability of the light source and environmental noise sources.

In the case of crossing the whole sensing loop, the sensing loop started to sense from the vicinity of the loop in both cases of vehicle and pedestrian. And it influenced the two optical fiber spools, simulating $12 \mathrm{~km}$-long sensor lines, although the fiber spools were isolated in shock absorbing boxes. The phase-sensitive $\Phi$-OTDR traces for these cases after averaging 32 times are shown in Figures 12 and 13. The vehicle driving over the sensing loop showed a broader peak signal at the $2 \mathrm{~km}$ location due to the averaging effect, as shown in Figure 12(b). However, the continuous walking motion by the pedestrian influenced not only the buried sensing loop, but also the two optical fiber spools, as seen in Figure 13(b). Also, the response by driving the vehicle $100 \mathrm{~m}$ away from the field test bed was observed in a similar pattern as the pedestrian walking over the sensing loop.

The appearance of the $\Phi$-OTDR trace was influenced by number of averaging times. Without averaging, it clearly showed a full swing variation at the impact location, wide in the case of vehicle and narrow in the case of pedestrian. This means phase changes of more than $2 \pi$ rad. in both cases. 


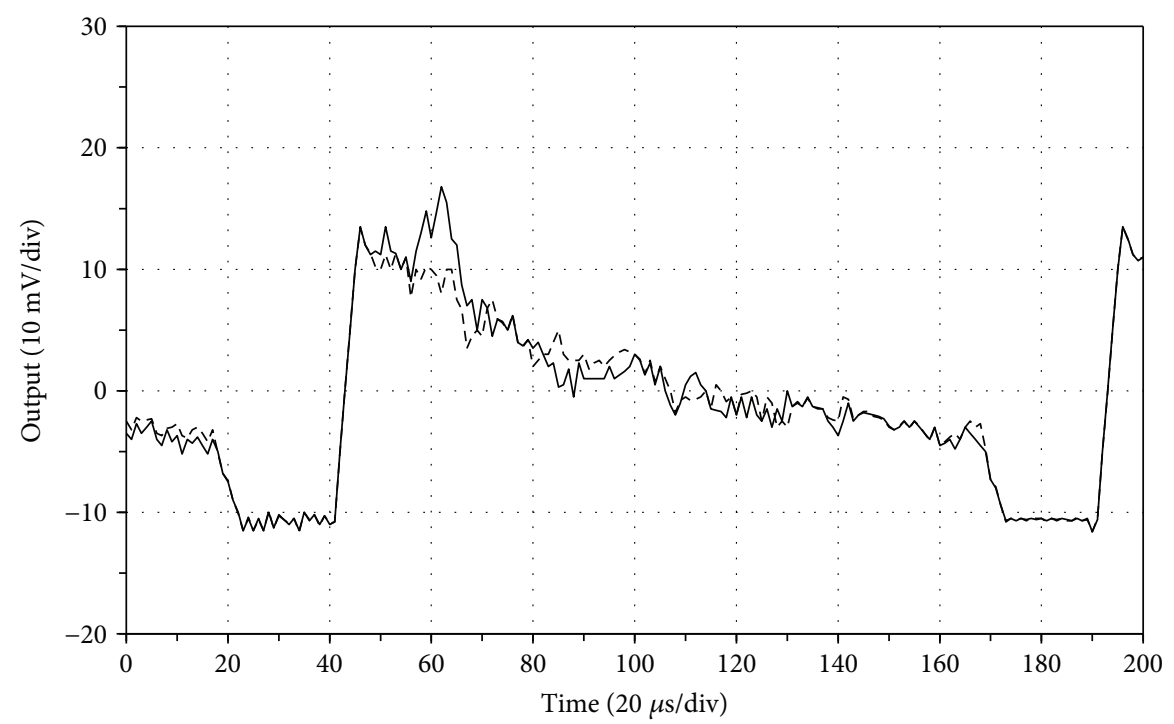

(a)

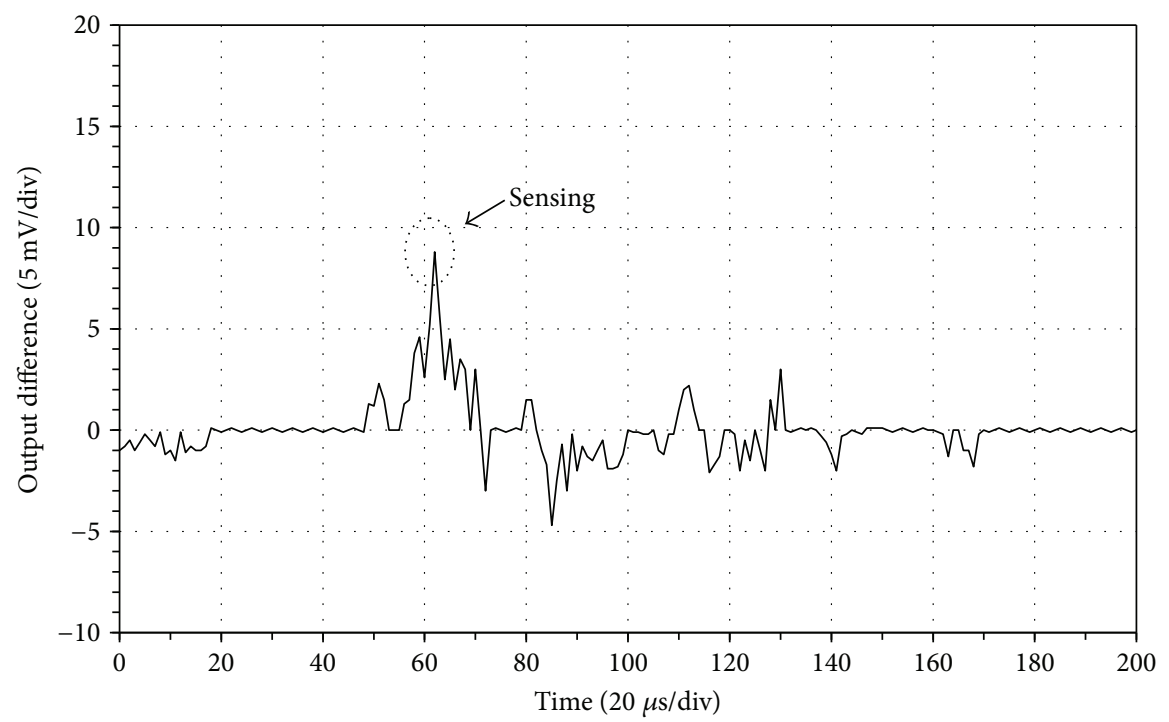

(b)

FIGURE 11: Phase-sensitive OTDR trace by pedestrian in case of crossing one sensing line. (a) Waveform before detection (dot) and after detection (line). (b) Difference waveform.

After averaging 256 times, the trace of the phase-sensitive $\Phi-O T D R$ response converged to a typical OTDR trace. The induced phase change is close to a linear function of applied weight for a given depth. In the case of the pedestrian weighing $60 \mathrm{~kg}$ over a sand box, the phase change is $6 \pi \mathrm{rad}$. at a burial depth of $20 \mathrm{~cm}$ and $2.5 \pi$ rad. at a burial depth of $40 \mathrm{~cm}$ according to the simulation results [27]. This implies that the phase changes by both vehicle and pedestrian are equal to or exceed $2 \pi \mathrm{rad}$. The sensitivity of this distributed optical fiber intrusion sensor was found to be too high to experimentally measure the distance resolution unless the entire $12 \mathrm{~km}$ sensing optical fiber was buried at a sufficient depth to cause less than $\pi / 2 \mathrm{rad}$. phase shift. However, the distance resolution can be inferred to be $\sim 160 \mathrm{~m}$ from a $3 \mathrm{~dB}$ width in Figure 11(b), when the light pulse width is $1.5 \mu \mathrm{s}$.
The spatial resolution of the system is proportional to the pulse width of incident light to optical fiber, as shown in (3), in the case where the backscattered light is sufficient enough for detection. And the shorter the length of this distributed sensor is, the larger the backscattered light is. Also, the larger the output power of the optical fiber amplifier is by cascading the other optical fiber amplifier, the larger the backscattered light is. Thus, the spatial resolution of the system is inversely proportional to the length of the sensing line. The minimum spatial resolution was measured as $\sim 40 \mathrm{~m}$ when the pulsed light output, with $0.3 \mu$ s pulse width, was amplified $+20 \mathrm{~dB}$ by cascading the second optical fiber amplifier for the $12 \mathrm{~km}$ sensing length.

The sensitivity of the system is a function of sensing length, burial depth, optical output power, and optical pulse width. By using the configuration in Figure 1 with 


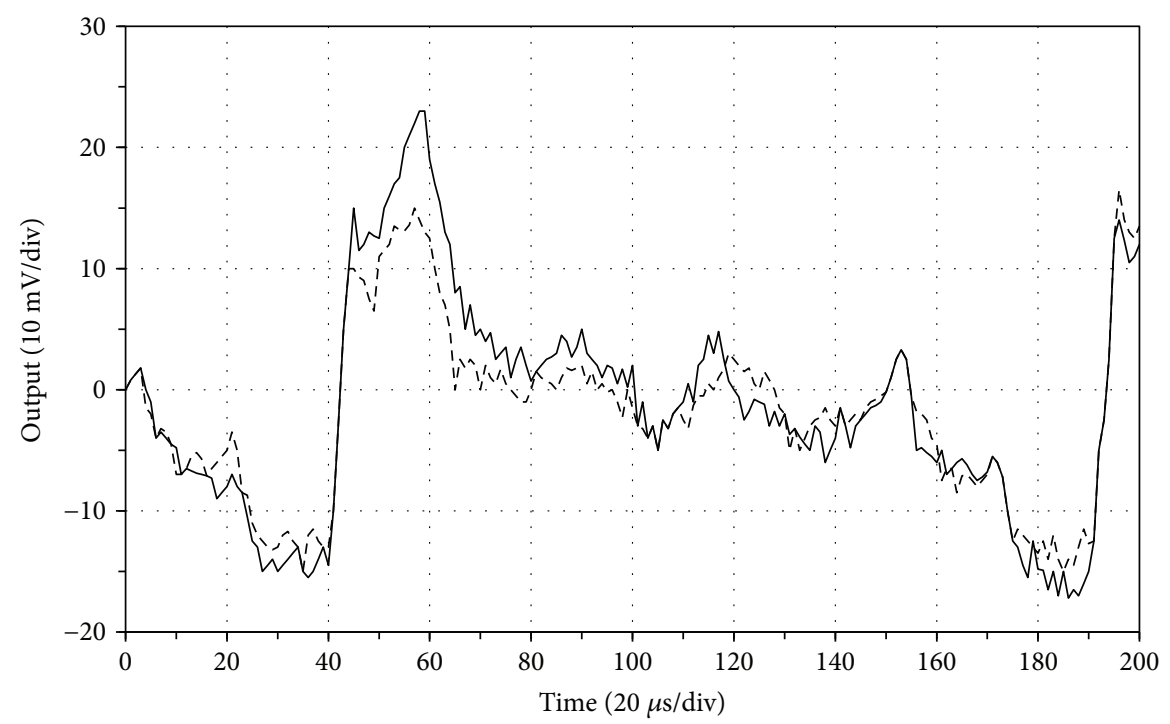

(a)

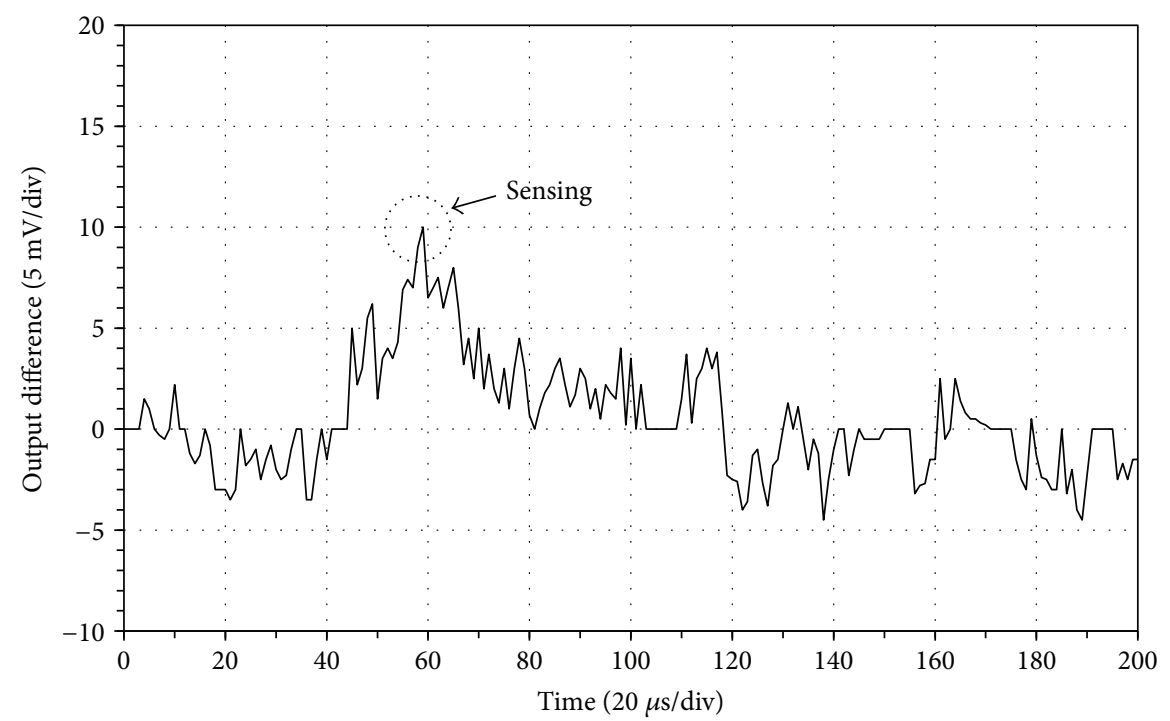

(b)

FIGURE 12: Phase-sensitive OTDR trace by vehicle in case of crossing whole sensing loop. (a) Waveform before detection (dot) and after detection (line). (b) Difference waveform.

the $12 \mathrm{~km}$ sensing line, adequate phase changes were produced for burial depths within $50 \mathrm{~cm}$ in the experiment. To predict the maximum sensing length, an analytical model using the Monte Carlo method was used by assuming the random locations of the scattering centers in the optical fiber. Twelve laser pulses were averaged for the signal level in each time bin. The number of missed intruders was determined in 100,000 trials. For a missed probability of $10^{-4}$ and a false alarm rate of $10^{-10}$, the sensing length was estimated as $50 \mathrm{~km}$ at a spatial resolution of $160 \mathrm{~m}$ by using the same parameter for spatial resolution measurement.

This distributed $\Phi$-OTDR intrusion sensor system is suitable for real-time monitoring of multiple intruders and the most cost-effective solution for long perimeter intrusion detection. And the coherent light source using the DFB laser diode with external optical feedback has the advantage of simpler architecture and is immune to environmental noises in comparison to other coherent light source using a fiber laser. The degree and stability of coherence length are critical to the performance of a $\Phi-O T D R$ intrusion sensor system. The coherence length variation due to environmental parameters fluctuates the phase of backscattered light, and this requires increasing the averaging number and further signal processing to reduce errors, resulting in a delayed response.

\section{Conclusion}

A distributed intrusion sensor using a DFB laser with external optical feedback and saturable absorber was fabricated for distributed intrusion sensor application. This light source had the advantage of a simple structure in comparison to a 


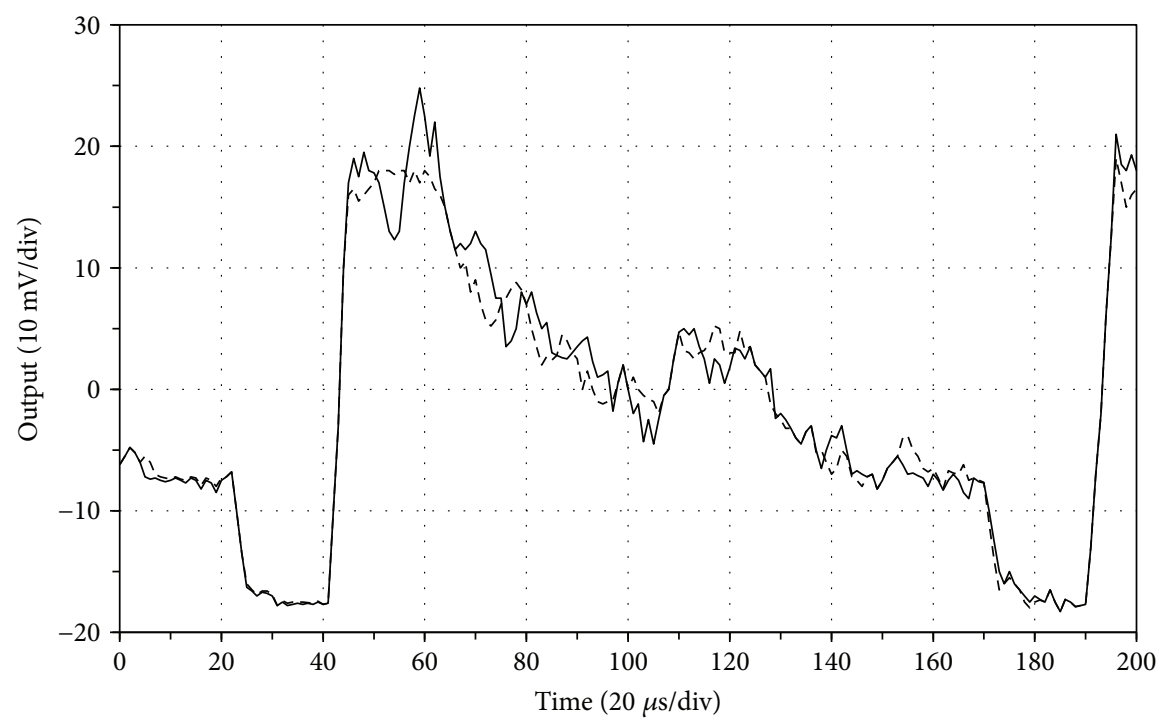

(a)

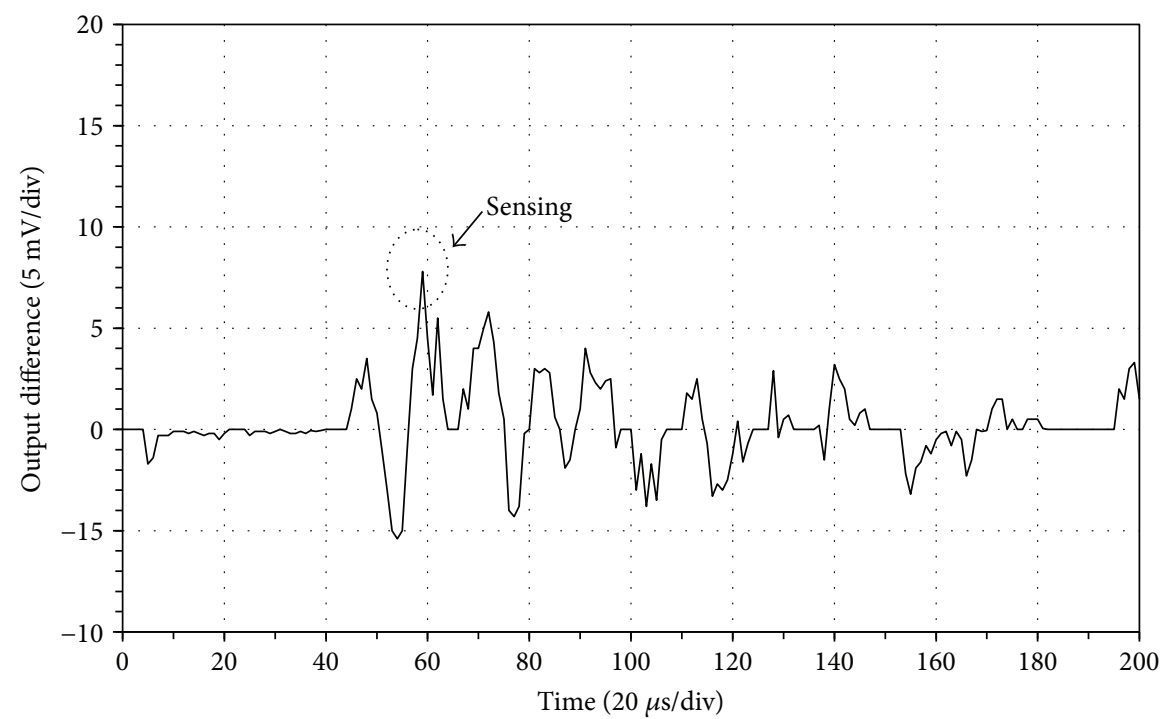

(b)

FIGURE 13: Phase-sensitive OTDR trace by pedestrian in case of crossing whole sensing loop. (a) Waveform before detection (dot) and after detection (line). (b) Difference waveform.

fiber laser, but with longer coherence time than a DFB laser alone. The linewidth of the light source was measured to be below the resolution limit of the self-heterodyne measurement setup, which is $\sim 3 \mathrm{kHz}$ FSR or $\sim 1 \times 10^{-8} \mathrm{~nm}$. The distributed intrusion sensor was simulated using two cascaded $2 \mathrm{~km}$ and $10 \mathrm{~km}$ fiber-optic spools. The intruder location was simulated at a location $2 \mathrm{~km}$ from the starting point. The distributed intrusion sensor in the laboratory setup showed sensible sensitivity to PZT stimulus. A field test was carried out by using two cascaded $2 \mathrm{~km}$ and $10 \mathrm{~km}$ fiberoptic spools. The intruder location was simulated at a location $2 \mathrm{~km}$ from the starting point by inserting a $100 \mathrm{~m}$ optical fiber with $3 \mathrm{~mm}$ diameter jacket. The test was carried out for two cases; one crossing the sensing loop and the other crossing one line only at a burial depth of $50 \mathrm{~cm}$. Two cases of intruders, a vehicle and a pedestrian, were attempted in the field experimental test. A single movement by the pedestrian across a sensing line or driving across a sensing loop by the vehicle induced the detection peaks at the $2 \mathrm{~km}$ intruder location. The induced phase changes by both intruders are considered to be above $2 \pi$ rad. from the measured responses. However, continuous movement by both the pedestrian walking and vehicle driving over a sensing loop induced multiple peaks around sensing peak due to the influence of optical fiber spools. The spatial resolution was measured as $\sim 40 \mathrm{~m}$ for the $12 \mathrm{~km}$ sensing length, and the usable sensitivity was found to be up to a burial depth of $50 \mathrm{~cm}$. The maximum sensing length was estimated to be up to $50 \mathrm{~km}$ at a spatial resolution of $160 \mathrm{~m}$ according to the simulation results. 


\section{Conflicts of Interest}

The author declares that there is no conflict of interests regarding the publication of this paper.

\section{Acknowledgments}

This work was supported by Incheon National University Research grant in 2016.

\section{References}

[1] S. Nagpal and P. Manojkumar, "Hardware implementation of intruder recognition in a farm through wireless sensor network," in 2016 International Conference on Emerging Trends in Engineering, Technology and Science (ICETETS), pp. 1-5, Tamil Nadu India, Febuary 2016.

[2] S. Yun, Q. Nguyen, and J. Choi, "Distributed sensor networks for multiple human recognition in indoor environment," in 2016 13th International Conference on Ubiquitous Robots and Ambient Intelligence (URAI), pp. 753-756, Xian China, August 2016.

[3] Y. Muanenda, C. Oton, T. Nannipieri, A. Signorini, S. Faralli, and F. Pasquale, "Hybrid distributed optical fiber sensors for temperature, strain and vibration measurement," in 2015 International Conference on Microwave, Optical and Communication Engineering (ICMOCE), pp. 1-4, Bhubaneswar, India, December 2015.

[4] Y. Wang, Z. Li, and Z. Jiang, “An improved distributed optical fiber sensor (DOFS) for monitoring long-distance buried oil pipeline leakage and intrusion," in 2009 4th IEEE Conference on Industrial Electronics and Applications, pp. 318-320, Xian, China, May 2009.

[5] J. C. Juarez and H. F. Taylor, "Field test of a distributed fiberoptic intrusion sensor system for long perimeters," Applied Optics, vol. 46, no. 11, pp. 1968-1971, 2007.

[6] J. Morosi, M. Mattarei, M. Ferrario, P. Boffi, and M. Martinelli, "Coherent fiber-optic sensor for vibration localization," in 2014 Fotonica AEIT Italian Conference on Photonics Technologies, pp. 1-4, Naples Italy, May 2014.

[7] K. Kajiwara, Z. He, and K. Hotate, "Distributed sensing inside long-length FBG at region beyond laser coherence length based on synthesis of optical coherence function," in CLEO:2011 - Laser Applications to Photonic Applications, pp. 1-2, Baltimore, MD, USA, May 2011.

[8] K. Harman, "Omnitrax ranging leaky coaxial cable sensor technology update," in 43rd Annual 2009 International Carnahan Conference on Security Technology, pp. 216-220, Zurich Switzerland, October 2009.

[9] M. Maki and D. Taylor, "CWD sensors for intrusion detection systems - an update," in Proceedings The Institute of Electrical and Electronics Engineers. 29th Annual 1995 International Carnahan Conference on Security Technology, pp. 317-324, Sanderstead England, October 1995.

[10] K. Choi, "Optical fiber speckle sensor for wire net fence application," in ICMIT 2005: Information Systems and Signal Processing, vol. 60412T, pp. 1-6, Chongqing, China, Febuary 2006.

[11] C. Bishop, T. Humble, R. Bennink, and B. Williams, "Intrusion detection based on quantum interference," in CLEO: Applications and Technology 2013, pp. 1-2, San Jose, CA, USA, June 2013.
[12] G. Allwood, G. Wild, and S. Hinckley, "Optical fiber sensors in physical intrusion detection systems: a review," IEEE Sensors Journal, vol. 16, no. 14, pp. 5497-5509, 2016.

[13] F. Feng, Z. Feng, X. Jia, Y. Rao, Z. Wang, and H. Wu, “128km fully-distributed high-sensitivity fiber-optic intrusion sensor with $15 \mathrm{~m}$ soatial resolution," in Optical Fiber Communication Conference 2014, pp. 1-3, San Francisco, CA, USA, March 2014.

[14] H. van de Hulst, Light Scattering by Small Particles, Dover Publications, New York, NY, USA, 1981.

[15] B. Crosignani, P. Porto, and M. Bertolotti, Statistical Properties of Scattered Light, Academic Press, New York, NY, USA, 1975.

[16] A. Hartog and M. Gold, "On the theory of backscattering in single-mode optical fibers," Journal of Lightwave Technology, vol. 2, no. 2, pp. 76-82, 1984.

[17] W. Davenport and W. Root, An Introduction to the Theory of Random Signals and Noise, McGraw-Hill, New York, NY, USA, 1958.

[18] S. Saito, O. Nilsson, and Y. Yamamoto, "Oscillation center frequency tuning, quantum FM noise, and direct frequency characteristics in external grating loaded semiconductor lasers," IEEE Journal of Quantum Electronics, vol. 18, no. 6, pp. 961-970, 1982.

[19] K. Iwatsuki, K. Hotate, and M. Higashiguchi, "Effect of Rayleigh backscattering in an optical passive ring-resonator gyro," Applied Optics, vol. 23, no. 21, pp. 3916-3924, 1984.

[20] M. W. Fleming and A. Mooradian, "Fundamental line broadening of single-mode (GaAl)As diode lasers," Applied Physics Letters, vol. 38, no. 7, pp. 511-513, 1981.

[21] Y. Yamamoto, S. Saito, and T. Mukai, "AM and FM quantum noise in semiconductor lasers- part II: comparison of theoretical and experimental results for AlGaAs lasers," IEEE Journal of Quantum Electronics, vol. 19, no. 1, pp. 47-58, 1983.

[22] K. Kojima, K. Kyuma, and T. Nakayama, "Analysis of the spectral linewidth of distributed feedback laser diodes," Journal of Lightwave Technology, vol. 3, no. 5, pp. 1048-1054, 1985.

[23] H. Ishii, K. Kasaya, and H. Oohashi, "Narrow spectral linewidth operation $(<160 \mathrm{kHz})$ in widely tunable distributed feedback laser array," Electronics Letters, vol. 46, no. 10, pp. 714-715, 2010.

[24] M. Fukuda, Optical Semiconductor Devices, John Wiley and Sons, New York, NY, USA, 1999.

[25] W. H. Loh, B. N. Samson, L. Dong, G. J. Cowle, and K. Hsu, "High performance single frequency fiber grating-based erbium:ytterbium-codoped fiber lasers," Journal of Lightwave Technology, vol. 16, no. 1, pp. 114-118, 1998.

[26] C. Akcay, P. Parrein, and J. P. Rolland, "Estimation of longitudinal resolution in optical coherence imaging," Applied Optics, vol. 41, no. 25, pp. 5256-5262, 2002.

[27] J. Park, Buried fiber optic sensor, [M.S. thesis], Texas A\&M University, College Station, TX, USA, 1992. 


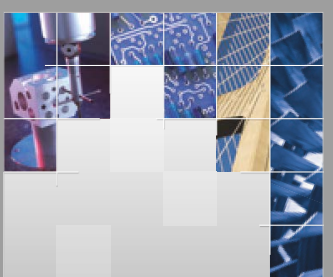

\section{Enfincering}
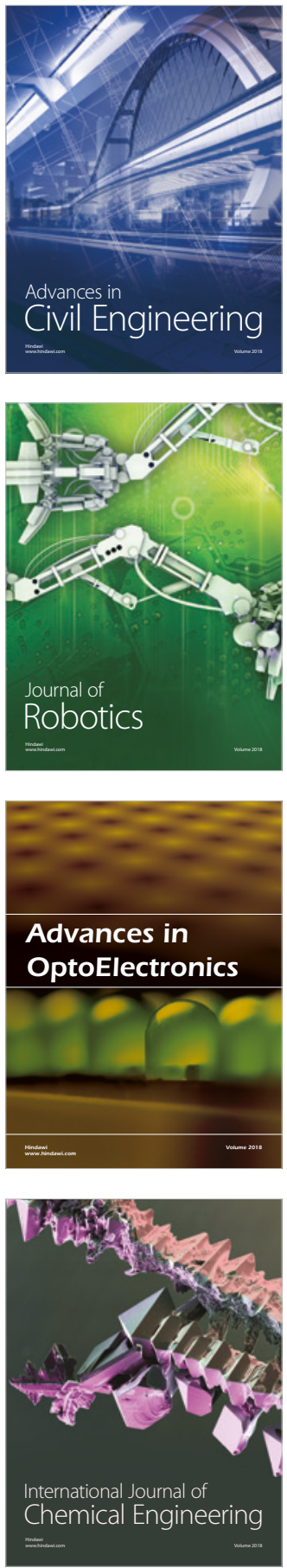

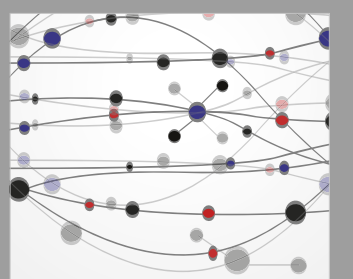

\section{Rotating \\ Machinery}

The Scientific World Journal

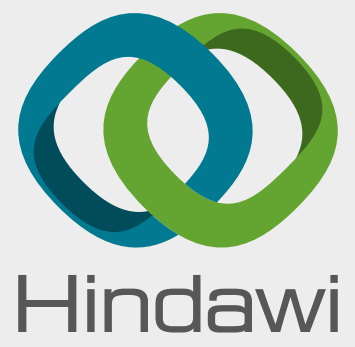

Submit your manuscripts at

www.hindawi.com
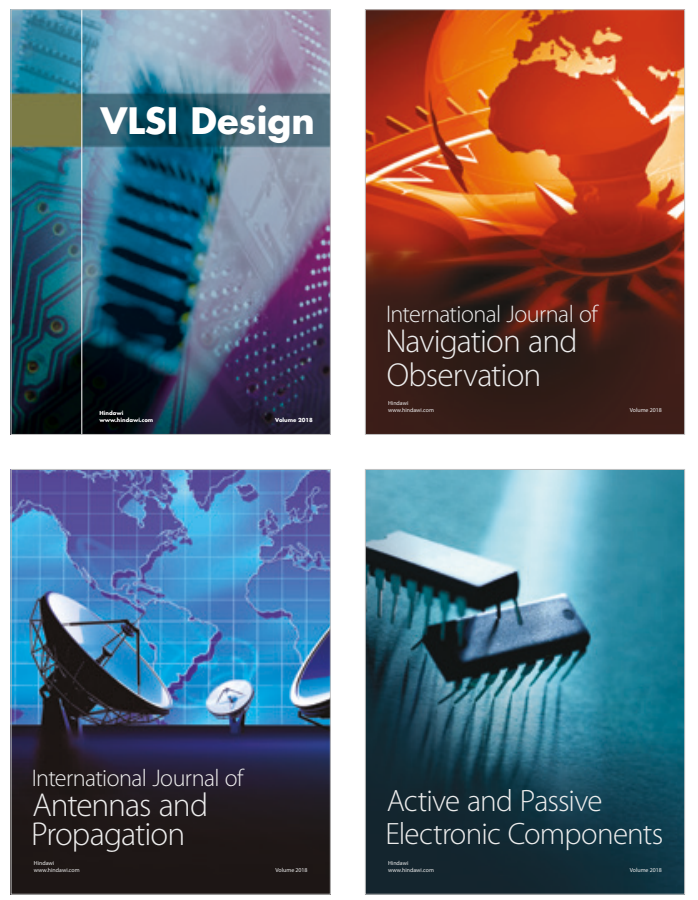
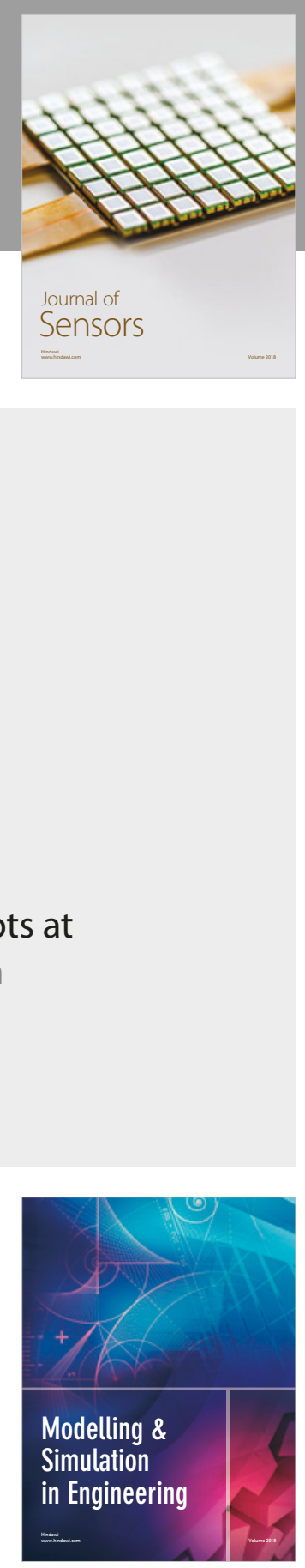

\section{Advances \\ Multimedia}
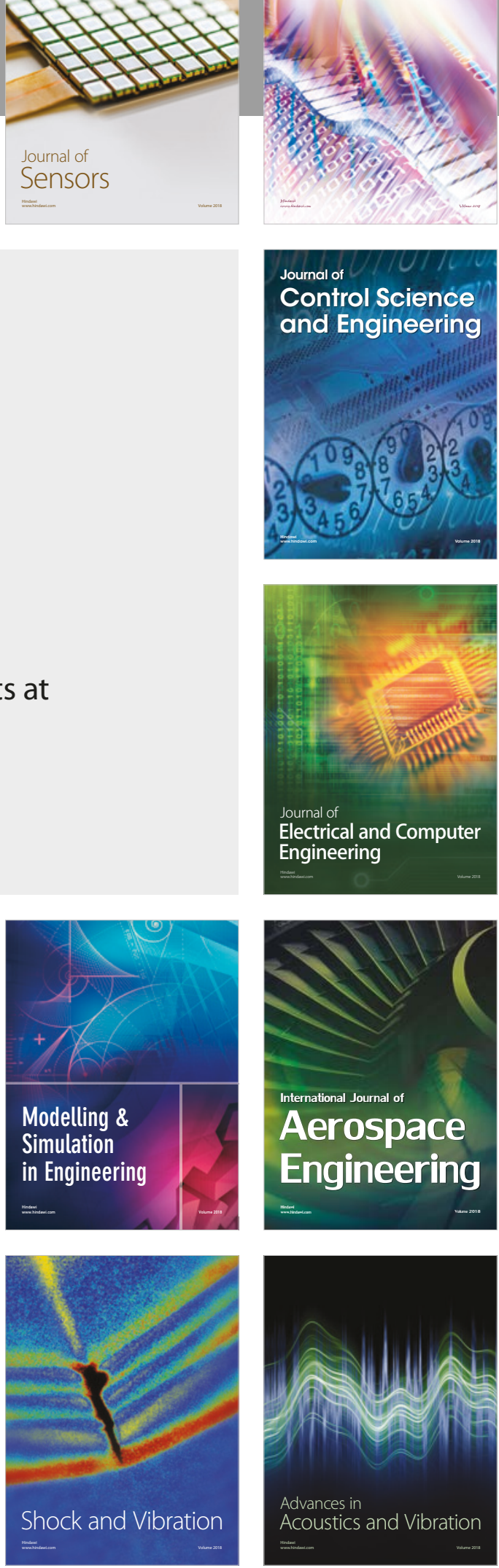